\title{
Neuronal insulin receptor substrate 2 (IRS2) expression is regulated by ZBP89 and SP1 binding to the IRS2 promoter
}

\author{
Michael Udelhoven, Mareike Pasieka, Uschi Leeser, Wilhelm Krone and Markus Schubert \\ Department of Internal Medicine II, Center for Molecular Medicine Cologne (CMMC) and Cologne Excellence Cluster on Cellular Stress Responses in \\ Aging-associated Diseases (CECAD), University of Cologne, Kerpener Straße 62, 50937 Cologne, Germany \\ (Correspondence should be addressed to M Schubert; Email: markus.schubert@uni-koeln.de)
}

\begin{abstract}
Since neuronal insulin receptor substrate 2 (IRS2)-mediated signals coordinate key processes in rodent physiology such as food intake, fertility, longevity, and aging-related behavior, we analyzed the mechanisms of neuronal IRS2 expression in neuroblastoma (SHSY5Y) and hypothalamic (GT1-7) cell lines. Using dual luciferase reporter assays and IRS2 promoter deletion constructs, we identified a regulatory cassette within the IRS2 promoter between -779 and $-679 \mathrm{bp}$ from the translational start which is responsible for $\sim 50 \%$ of neuronal IRS2 promoter activity. Chromatin immunoprecipitation assays and electromobility shift assay revealed four overlapping ZBP89/specificity protein 1 (SP1) binding sites which alternatively bind to ZBP89 (ZNF148 as listed in the HUGO Database) or SP1. Activation of this cassette is inhibited by
\end{abstract}

phosphoinositide-3-kinase (PI3K) via increased ZBP89 binding to the promoter. Serum starvation caused increased SP1 binding at one specific SP1 site and decreased binding to another, proving a regulatory interaction between the different binding sites within this promoter cassette to tightly control IRS2 expression. Mutants containing all the possible combinations of one, two, three, or all the four SP1 binding sites of the IRS2 promoter revealed that SP1 binding to one particular site is most important for promoter activation. Stable downregulation of ZBP89 using siRNA substantially increased IRS2 mRNA and protein expression. Thus, alternative binding of ZBP89 or SP1 to the described region in the IRS2 promoter regulates neuronal IRS2 expression in a PI3K-dependent manner.

Journal of Endocrinology (2010) 204, 199-208

\section{Introduction}

The insulin receptor substrate (IRS) protein family has at least four members, IRS1 to IRS4 (Sun et al. 1991, 1995, Lavan et al. 1997a,b), mediating the intracellular effects of the insulin and insulin-like growth factor 1 (IGF1) receptors. IRS protein-transmitted signals are involved in the regulation of cellular key processes such as transcription, protein synthesis, proliferation, and apoptosis (White 2003). Upon activation, the IRS proteins bind to several Src homology $(\mathrm{SH})_{2}$ domaincontaining signaling proteins. This leads to the activation of several downstream targets, such as phosphoinositidedependent protein kinase-1, protein kinase B (PKB/AKT), p70 ${ }^{\text {S6kinase }}$, and GRB2, and inhibition of glycogen synthase kinase-3 as well as to the activation of the MAP-kinase pathway (White 2003). The function of the different IRS proteins has been studied extensively in peripheral tissues such as muscle, liver, and pancreas (Thirone et al. 2006, Haeusler \& Accili 2008, Kubota et al. 2008). IRS2 has been shown to mediate insulin's effect to suppress hepatic gluconeogenesis and pancreatic $\beta$-cell compensation to insulin resistance (Withers et al. 1999, Dong et al. 2008). Consequently, investigation on IRS2 transcription has been focused on these tissues. The deepest insight into the regulation of IRS2 transcription has been gained from studies in hepatocytes. Briefly, insulin is able to downregulate IRS2 promoter activity in rat hepatocytes (Zhang et al. 2001). Zhang et al. $(2001,2003)$ found a heptanucleotide in the IRS2 promoter functioning as insulin-responsive element. Furthermore, sterol regulatory element binding proteins (SREBPs) are able to bind to the IRS2 promoter and suppress IRS2-mediated signaling in the liver. The forkhead transcription factor FKHRL1 (FOXO3) induces IRS2 transcription, which is suppressed by SREBP-1a and $-1 c$ overexpression (Ide et al. 2004, Shimano 2007). PPAR $\gamma$ coactivator-1 activates the IRS2 promoter, and this effect is inhibited by SREBP-1 as well. In the same region of the IRS2 promoter, there is an E-box overlapping the SREBP-1 and neighboring the FKHRL1 binding site. TFE3 (TCFE3 as listed in the HUGO Database) binds to this E-box to activate IRS2 promoter synergistically with the forkhead transcription factor (Fox)O1 (Nakagawa et al. 2006).

So far, there is no information on the mechanisms of Irs gene transcription in neurons available even though Irs 1, 2, and 4 are expressed in rodent brain. Irs 4 expression is restricted to the hypothalamus, whereas Irs 1 and 2 are found throughout the CNS. Recent data suggest that neuronal Irs 2 expression exhibits distinct function during brain development, in metabolic regulation, and in rodent fertility 
(Burks et al. 2000, Schubert et al. 2003). Most recent reports suggest that IRS2 in the CNS regulates aging-related behavior and longevity. In particular, IRS2 deficiency in mice causes decreased neuronal proliferation and decreased fertility. Neuron-specific deletion of IRS2 prolongs lifespan (Taguchi et al. 2007), and hypothalamic IRS2 deficiency leads to increased food intake and obesity (Lin et al. 2004). Furthermore, neuronal IR (INSR)/IGF1/IRS2-mediated signals might be involved in the pathogenesis of human neurodegenerative diseases. Postmortem investigations of brains from patients with Alzheimer's disease revealed a markedly downregulated expression of IR and IGF1R as well as of IRS2, and these changes progressed with the severity of neurodegeneration, suggesting a role of IRS2 expression in the pathogenesis of neurodegenerative diseases (Rivera et al. 2005, Steen et al. 2005).

Since neuronal IRS2 might be involved in the development of metabolic disorders and possibly neurodegenerative diseases, we aimed to identify mechanisms regulating the transcription of IRS2 in neurons. Currently, there are no published studies available dealing with regulatory mechanisms underlying transcription of the IRS2 gene in neurons. In the current study, we demonstrate that alternative binding of ZBP89 (ZNF148 as listed in the HUGO Database) or specificity protein 1 (SP1) to a regulatory cassette in the IRS2 promoter regulates neuronal IRS2 expression in a phosphoinositide-3kinase (PI3K)-dependent manner.

\section{Materials and Methods}

\section{Chromatin immunoprecipitation assay}

SHSY5Y cells were seeded in 10-cm dishes. One plate was used per experiment. Cells were kept serum free for $30 \mathrm{~h}$, and were fixed with $1 \%$ formaldehyde for 10 and 5 min with $125 \mathrm{mM}$ glycine at reverse transcription (RT). Cells were washed and harvested in $2 \mathrm{ml}$ PBS. Pellets were lysed in lysis buffer $(5 \mathrm{mM}$ PIPES, pH 8, $85 \mathrm{mM} \mathrm{KCl,} \mathrm{0.5 \%} \mathrm{NP40,} \mathrm{and} 1 \times$ complete protease inhibitor (Roche)), and plasma membranes were destroyed by shearing through a 26 gauge needle. After $10 \mathrm{~min}$ on ice, nuclei and cell debris were pelleted at $1000 \mathrm{~g}$. After resuspending in $750 \mu$ high salt lysis buffer $(1 \times$ PBS, $1 \%$ NP40, $0 \cdot 5 \%$ sodium deoxycholate, $0 \cdot 1 \%$ SDS, and $1 \times$ complete protease inhibitor) to open the nuclei, lysates were left on ice for $10 \mathrm{~min}$. Released DNA was sonicated four times for $30 \mathrm{~s}$ at $50 \%$ power using a sonicator (Sonoplus-M73, BandelinElectronic, Berlin, Germany) on ice. Remaining cell debris was pelleted at $11000 \mathrm{~g}$, and supernatant was used for immunoprecipitation. Samples were split, and $350 \mu \mathrm{l}$ were incubated with a specific antibody $(\mathrm{ab} ; 3 \mu \mathrm{g})$ (anti-SP1 (H-225) X, anti-ZBP89 (H-184) X (Santa Cruz Biotechnology, Santa Cruz, CA, USA), and anti-RNApol II \#39097 (Active Motif, Carlsbad, CA, USA) and $350 \mu$ with a nonspecific control ab (3 $\mu \mathrm{g}$ ) (normal mouse IgG \#2025, normal rabbit IgG \#2027 (Santa Cruz Biotechnology)), and $50 \mu \mathrm{l}$ were left for input control. After incubating with the antibody (ab) for $4 \mathrm{~h}$ at $4{ }^{\circ} \mathrm{C}$ on a shaker, blocked protein $G$ agarose was added and incubation was continued overnight. Prior to usage, protein $\mathrm{G}$ agarose was blocked in 1\% BSA with $50 \mu \mathrm{g}$ salmon sperm DNA (Sigma) in high salt lysis buffer. The next day, beads were washed twice with lysis buffer and four times with a wash buffer $(100 \mathrm{mM}$ Tris-HCl, pH 8, $500 \mathrm{mM} \mathrm{LiCl,} \mathrm{1 \%} \mathrm{NP40,}$ and $1 \%$ sodium deoxycholate). After that, ab-coupled protein was released by incubation in $150 \mu$ l elution buffer $(1 \%$ SDS and $0 \cdot 1 \mathrm{M} \mathrm{NaHCO}_{3}$ ) for $1 \mathrm{~h}$ at $25{ }^{\circ} \mathrm{C}$. Agarose was pelleted and $\mathrm{NaCl}$ was added to the supernatant to a final concentration of $250 \mathrm{mM}$. Previously taken input controls were treated similarly. RNase A and proteinase $\mathrm{K}$ were added, and samples were incubated at $67^{\circ} \mathrm{C}$ overnight. After decrosslinking, DNA was cleaned up with PCR cleanup kit (Qiagen).

\section{PCR in chromatin immunoprecipitation assay}

PCR for chromatin immunoprecipitation (ChIP) assay was performed using $5 \mu \mathrm{l}$ of eluted DNA per sample. PCR was performed with a standard 30-cycle PCR program using $55^{\circ} \mathrm{C}$ as the annealing temperature and $72{ }^{\circ} \mathrm{C}$ as the elongation temperature. Elongation time was $45 \mathrm{~s}$, and Taq Polymerase LC (Fermentas Int. Inc., Burlington, Ontario Canada) was used in $1.5 \mathrm{mM} \mathrm{MgCl} 2$ containing PCR buffer. Primers for ChIP PCR were 5'-ACAAGCCGCTGATTAATGAGGC-3', 5'-TGACTCGGCGTTACGCAGGCAC-3' for IRS2 promoter and 5'-TACTAGCGGTTTTACGGGCG-3', 5'-CGAACAGGAGGAGCAGAGAGCGA-3' for Gapdh promoter. PCR samples were separated on a $1 \cdot 5 \%$ agarose gel in TAE buffer.

\section{Cloning of IRS2 promoter luciferase reporter constructs}

Full-length $-2338 \mathrm{bp}$-IRS2 promoter was amplified from human genomic DNA using the following primers: $5^{\prime}$-CCCAAATTCAGGCTACTGATCAATAATCC-3', 5'-CCCACCGCTGCAGGAGCACC-3', 5'-GAAGGGGGTGCTCCTGCAGCGGTG-3', and $5^{\prime}$-AAAAAGCTTCGCGGGCGCTTCAGGCCGCG-3' ${ }^{\prime}$. The resulting two PCR subfragments were cloned into pDrive (Qiagen) and pCR2.1 (Invitrogen). To construct the full-length promoter, the fragments were ligated XhoI/PstI and PstI/HindIII into pGL2 basic (Promega). IRS2-1585 bp was cut XhoI/HindIII out of IRS2-1585 bp-pCR2.1 and ligated XhoI/HindIII into pGL2. The other IRS2 promoter fragments $-1962,-1235,-824,-688,-611,-500$, and -398 bp were constructed using $5^{\prime}$-AAACTCGAGGCGCAGGATCGGGAGCTTGCTG-3', 5'-AACTCGAGCTCGATCTGGAGCGCGGTGCTCGC-3', 5'-AACTCGGACAAGCCGCTGATTAATGAGGC-3', $5^{\prime}$-AACTCGAGCCGCGAGCGCCGCGCCGATTGGCCGAGC-3', 5'AACTCGAGCGGCCGCGCTGTGTGTGCCTGCGTAA-3', 5'-AACTCGAGAGCCCGGGTCGCCGTTGGCAGC- $3^{\prime}$, 5'-ACTCGAGAGCGCGACGCGGCGGCAGCAGC- $3^{\prime}$ as the forward primers and $5^{\prime}$-AAAGCTTCGCGGGCGCTTCAGGCCGCG- $3^{\prime}$ as the reverse 
primer. IRS2 promoter full-length clone was used as a template, and fragments were ligated XhoI/HindIII into pGL2.

\section{Dual luciferase reporter assay}

SHSY5Y cells as well as GT1-7 cells were transfected with $0.5 \mu \mathrm{g}$ promoter reporter construct and $10 \mathrm{ng}$ pRLCMV (Promega) per well in 24-well plates (Greiner Bio-One, Frickenhausen, Germany) using Effectene (Qiagen) according to the manufacturers' instructions. All measurements were done in triplicates $(n=6$ independent experiments). After seeding in DMEM with 10\% FCS and penicillin/streptomycin, cells were transfected the day after. After starving the cells in DMEM without FCS for $30 \mathrm{~h}$, dual luciferase reporter assay was performed according to the manufacturers' instructions (Promega) using a Mithras LB940 luminometer (Berthold Technologies, Bad Wildbad, Germany).

\section{Electromobility shift assay}

Nuclear extracts (NEs) were prepared from SHSY5Y cells that were starved in DMEM without FCS for $30 \mathrm{~h}$. All procedures were performed at $4{ }^{\circ} \mathrm{C}$. Cells, $10^{7}$, were pelleted, and lysed as described previously (Dignam et al. 1983). Protein concentration of the final supernatant was determined by Bradford assay. Totally, $8 \mu \mathrm{g}$ NE was used per sample. Oligonucleotides used in electromobility shift assay (EMSA) were annealed to yield double strands in $10 \mathrm{mM}$ Tris- $\mathrm{HCl}, \mathrm{pH}$, and $50 \mathrm{mM} \mathrm{NaCl}$, and the binding reaction was performed using the LightShift Chemiluminescent EMSA kit (Pierce). Oligonucleotides used for NF1 and SP1 binding were site A: $5^{\prime}$-CCCGCCCGGCCGGGC CGGGGCCTTCGCGGGAG-3' and $5^{\prime}$-CTCCCGCGAAGGCCCCGGCCCGG CCGGGCGGG-3', site B: 5'-GGCCTTCGCGGGAGGGGGAGGGGACGGCGGGA-3 $3^{\prime}$ and $5^{\prime}$-TCCCG CCGTCCCCTCCCCCTCCCGCGAAGGCC-3', site C: 5'-GGCCCGGGGAGAAAGGGGGGCGGGGCGGGGGCGC-3' and $5^{\prime}$-GCGCCCCCGCCCCGCCCCCCTTTCTCCCCGGGCC-3' ${ }^{\prime}$, and site D: 5'-GGGGC GCGCGGCCCGCCCCCGCGAGCGCCGCGCC-3' and $5^{\prime}$-GGCGCGGCGCTCGCGGGGGCGGGCCGCGCGCCCC-3'. For supershift, anti-SP1 (H-225) X and anti-ZBP89 (H-184) X (Santa Cruz Biotechnology) were used. To quantify changes in optical density of supershift bands, the software AIDA (Version 4.00.027, Raytest, Straubenhardt, Germany) was used.

\section{Western blot}

Antibodies used for western blotting after SDS-PAGE were anti-IRS2 \#4502, anti-AKT \#9272, anti-phospho AKT
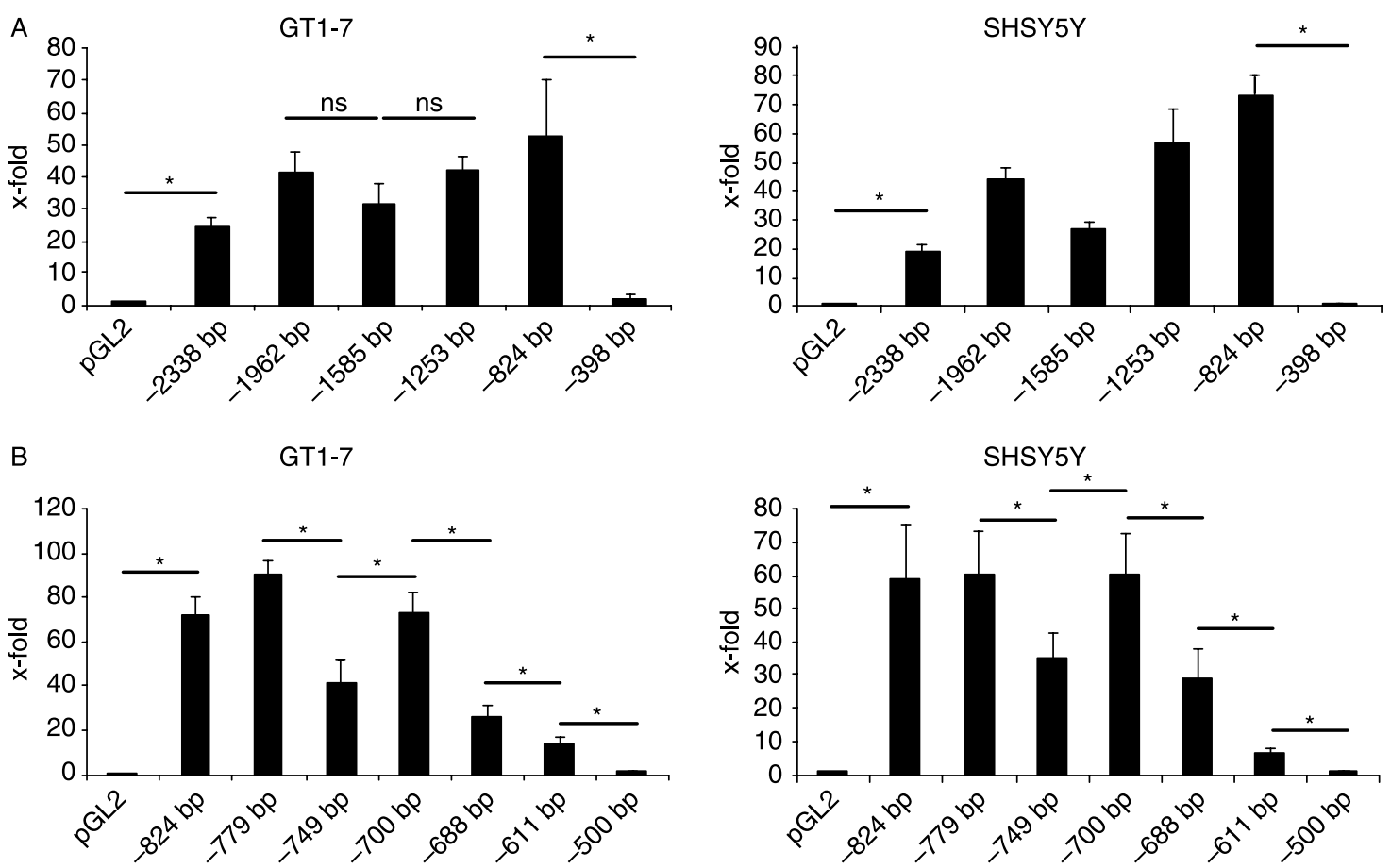

Figure 1 Isolation of the main activating regions in the IRS2 promoter using promoter deletion constructs. Dual luciferase assays were performed in GT1-7 and SHSY5Y cells using IRS2 promoter deletion constructs shortened stepwise from upstream. Cells were kept under serum-free conditions for $30 \mathrm{~h}$ before measurement. (A) IRS2 promoter fragments from -2338 to -398 bp were tested for luciferase activity. (B) Fragments from -824 to -500 bp were used for sublocalization. Quantification represents six independent experiments. Data are means \pm s.D. $\left({ }^{*} P<0 \cdot 0005\right.$, unpaired Student's $t$-test). 
(Ser473) \#9271 (Cell Signaling Technology, Inc., Danvers, MA, USA), anti-ZBP89 (H-184) (Santa Cruz Biotechnology), and anti-actin C4 (MP Biomedicals, Solon, OH, USA).

\section{Site-directed mutagenesis}

SP1 binding sites on -824 bp-IRS2 pGL2 were mutated individually and after that in sequential order to achieve double, triple, and complete mutations of all the SP1 binding sites. Mutagenesis was performed using primers $5^{\prime}-\mathrm{A}$ CCCCGCCCGGCCGGGAAAAGGCCTTCGCGGGAGGGGGAGGGGACGGC-3' and 5'-GC CGTCCCCTCCCCCTCCCGCGAAGGCCTTTTCCCGGCCGGGCGGGGT-3' in the case of site A, $5^{\prime}$-CGGGGCCTTCGCGGGAGGGGGAAAAG ACGGCGGGAAACGCGGCCCG-3' and $5^{\prime}$-CGGGCC GCGTTTCCCGCCGTCTTTTCCCCCTCCCGCGAAGGCCCCG-3' in the case of site B, 5'-GAAACGCG GCCCGGGGAGAAAGGGGGGAAAAGCGGGGGCGCGCG-3' and $5^{\prime}$-CGCGCGCCCCCGCTTTTCCCCCCTTTCTCCCCGGGCCGCGTTTC- $3^{\prime}$ in case of site $\mathrm{C}$, and 5'-CGGGGGCGCGCGGTTTTCCCCCGCGAGCGCCGCGCCGATTGGCC-3' ${ }^{\prime}$ and $5^{\prime}$-GGCCAATCGGCGC-
GGCGCTCGCGGGGGAAAACCGCGCGCCCCCG-3' in case of site $\mathrm{D}$. The single mutants were prepared using IRS2-824 bp-pGL2 as a template. PCR was performed with Hifi Taq Proofreading Polymerase (Fermentas) for $45 \mathrm{~s}$ at $60{ }^{\circ} \mathrm{C}$ and for $8 \mathrm{~min}$ at $72{ }^{\circ} \mathrm{C}, 32$ cycles. DNA was digested with DpnI, and the religated fragments were transformed into Escherichia coli.

\section{Quantitative real-time $R T-P C R$}

Total RNA of $1.5 \mu \mathrm{g}$ extracted from SHSY5Y wild-type and stable transfected cells was converted to cDNA using Superscript II RT (Invitrogen) with random primer protocol according to the manufacturers' instructions. Real-time PCR samples were prepared using Taqman Gene Expression Master mix and human-specific Taqman Gene Expression assays (Applied Biosystems, USA) for IRS2 (Hs00275843_s1 IRS2 FAM) with endogenous control for human TBP (4326322E VIC) according to the manufacturers' instructions. Real-time PCR was performed on a Step One Real-Time PCR System (Applied Biosystems). Data were analyzed and quantified using the $\Delta \Delta C_{\mathrm{t}}$ method (Pfaffl 2001).
A

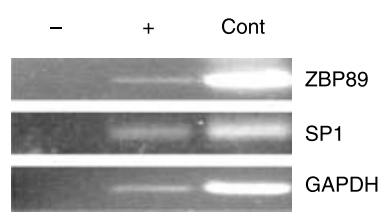

B

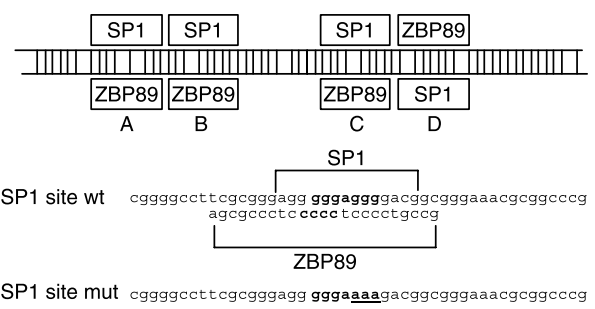

C

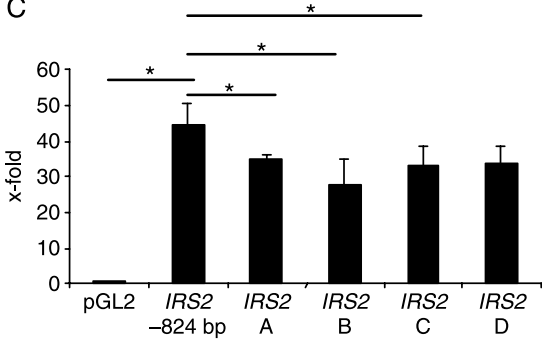

F

$$
\begin{aligned}
& \text { Site A geccggecgggecggggcct } \\
& \text { Site B cgcgggagggggaggggacg }
\end{aligned}
$$

Figure 2 Verification of SP1 and ZBP89 binding and activation of the single-, double-, triple-, and fourfold mutated Irs2 promoter regulatory cassette in SHSY5Y cells. (A) For verification of binding in ChIP assays SHSY5Y cells were fixated with formaldehyde, nuclear DNA was prepared, and after sonication fragmented and crosslinked DNA was incubated with antibodies against ZBP89, SP1, or RNApol II. After immunoprecipitation, decrosslinking, and DNA purification, the postulated binding region was localized via specific PCR primers. GAPDH primers were used for RNApol II control reaction. IP was performed using unspecific antibodies (-) of the same isotype and specific antibodies (+) against ZBP89, SP1, or RNApol II. Total sonicated decrosslinked DNA was used as positive input control (cont) for PCR. SP1 binding sites were mutated using site-directed mutagenesis as described in experimental procedures. The potential core ZBP89 binding sites as shown in (B) remained intact after mutagenesis. For dual luciferase reporter assays, SHSY5Y cells were kept serum free for $30 \mathrm{~h}$ before measurement. SHSY5Y cells were transfected with single (C), double (D), and triple or quadruple mutations (E) of the SP1 binding sites within the IRS2 promoter regulatory cassette located on the -824 bp-IRS2 pGL2 construct. Quantification represents six independent experiments. Data are means \pm S.D. $\left({ }^{*} P<0 \cdot 0005\right.$, unpaired Student's $t$-test). (F) Promoter regulatory cassette regions A-D. Core binding sites are underlined. 
Construction of stable transfected cell lines

SHSY5Y cells were transfected with $2 \mu \mathrm{g}$ linearized vectorconstruct (in the case of pCMV-FLAG, mIRS2 vector was cut with MluI, if pSuppressor Neo was used, the vector was cut with EcoRV (Fermentas)) with Effectene (Qiagen) according to the manufacturers' instructions. Two days after the transfection, cells were treated with a selection medium containing $1 \mathrm{mg} / \mathrm{ml} \mathrm{G} 418$ (Sigma). After 3 weeks of selection, cells were harvested for separation. These clones were checked for expression, and single cells were picked. Clones grown from these single cells were tested for expression, and positive clones were selected for further experiments. Cells used for the experiments were grown without G418.

\section{Results}

\section{IRS2 promoter activity in SHSY5Y and GT1-7 cells}

To perform dual luciferase reporter assays in SHSY5Y and GT1-7 cells, which provide model systems for neuronal cells, we cloned the full-length promoter of IRS2 (2338 bp). After sequence confirmation, the promoter was cloned into pGL2. The full-length promoter showed high basal luciferase activity compared to mock transfection proving that the cloned sequence is a functional promoter (data not shown). To identify new transcription factors binding to and/or modulating IRS2 promoter activity, deletion constructs getting shorter from upstream in steps of $300 \mathrm{bp}$ were amplified using PCR (Fig. 1A and B). Promoter activity was nearly abolished using deletion constructs shorter than -824 bp in GT1-7 and SHSY5Y cells suggesting a major regulatory element proximal to $-824 \mathrm{bp}$ from the translation start site. To analyze this region of the IRS 2 promoter in detail, we created further deletion constructs getting shorter from $-824 \mathrm{bp}$. Interestingly, there was no simple breakdown of promoter activity after stepwise cutting down to $-500 \mathrm{bp}$. In fact, basal luciferase activity of the different deletion constructs getting successively shorter went up and down, arousing suspicion of the existence of an activation cassette with multiple transcription factor binding sites. In detail, promoter activity decreased after cutting down to $-749 \mathrm{bp}$ and rose again after shortening to $-700 \mathrm{bp}$ and decreased stepwise after shortening to $-688,-611$, and finally to $-500 \mathrm{bp}$ (Fig. 1B). After sequence comparison with a transcription factor binding site database (MatInspector, www.genomatix.de), we proposed an activation cassette of four ZBP89 (Wang et al. 1993) binding sites overlapping with four SP1 binding sites (Fig. 2D and F) between -779 and -679 bp upstream of the translation start site.

\section{Confirmation of ZBP89 and SP1 binding to the activation cassette by ChIP assay}

We performed ChIP assays using primers designed to amplify the whole activation cassette. ChIP assay was carried out as described in Materials and Methods. Binding of ZBP89 and SP1 to the promoter was validated via positive PCR signals precipitated by specific antibodies (Fig. 2A). There was no signal detectable in control PCR using unspecific antibodies. Positive control for sonicated genomic DNA is shown in the right column of Fig. 2A. ChIP assay with GAPDH primers as control is shown at the bottom in Fig. 2A proving functionality of the assay.
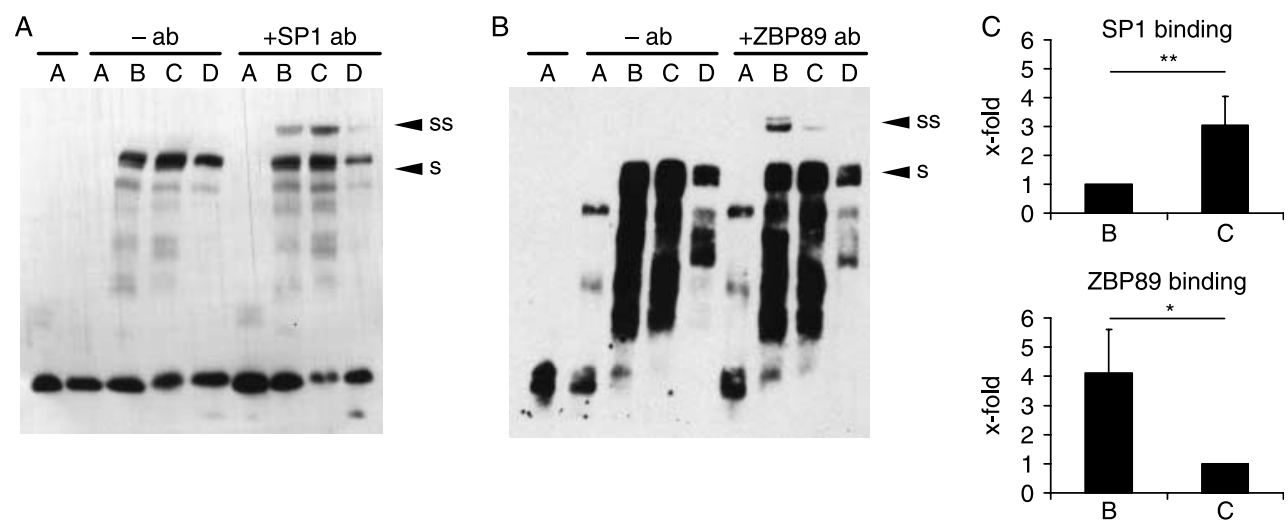

Figure 3 Differential binding of SP1 and ZBP89 to the IRS2 promoter. (A) NEs from cells kept without serum for $30 \mathrm{~h}$ were incubated without antibody but with biotinylated double-stranded oligonucleotides representing binding sites A, B, C, and D respectively (lanes 2-5). Lanes 6-8 represent the same approach, but were incubated with SP1 antibodies for supershift. Biotinylated double-stranded oligonucleotides representing binding site $A$ (lane 1) were incubated without NEs for control. (B) The same experiment as in (A) is shown, but ZBP89 antibodies were used for supershift. Shift and supershift bands are indicated by arrows (C) Quantification of SP1/ZBP89 binding to sites B and C using optical density of supershift bands. Data are means \pm s.D. $\left({ }^{* *} P<0 \cdot 0005,{ }^{*} P<0 \cdot 02\right.$, unpaired Student's $t$-test). Quantification represents seven independent experiments. 
The regulatory cassette of the IRS2 promoter in neuronal cells consists of four overlapping ZBP89/SP1 binding sites

Sequence comparison using MatInspector revealed binding sites for ZBP89 and SP1. Both transcription factor binding sites overlap, as it has been shown in other promoters (Law et al. 1998, Merchant et al. 1999, Wieczorek et al. 2000) with SP1 as the activator and ZBP89 as the repressor. The first part of the activation cassette starts with binding site A $(-779$ to $-766 \mathrm{bp})$ followed directly by site B $(-765$ to $-743 \mathrm{bp}$ ). The potential SP1 binding sites are adjacent on the same side of the DNA helix. Potential ZBP89 binding sites overlap in sequence with SP1 sites. The second part of the activation cassette consisting of binding sites $\mathrm{C}(-726$ to $-704 \mathrm{bp})$ and $\mathrm{D}(-701$ to $-679 \mathrm{bp})$ lies $20 \mathrm{bp}$ apart. Here, the potential binding sites of ZBP89 and SP1 are arranged crossover as shown in Fig. 2B.

\section{Binding site B is most important for promoter activation in neuronal cells}

We generated IRS2 luciferase promoter constructs containing all the possible combinations of one, two, three, or all the four SP1 binding sites mutated (Fig. 2C-E). We found that promoter activity was decreased if binding site B (between -779 and -688 bp from translation start) was mutated alone or in combination with the other SP1 binding sites (Fig. 2C-E). Mutation of all the four SP1 binding sites (A, B, C, D, Fig. 2E) resulted in about $50 \%$ decreased promoter activity.

Binding sites $A, B, C, D$ show different preference to ZBP89 and SP1 in EMSA

Detailed analyses of all the four ZBP89/SP1 binding sites revealed a preference of ZBP89 binding to site $\mathrm{B}$ and a predominant binding of SP1 to site $\mathrm{C}$ in EMSA experiments (Fig. 3A and B, Supplementary Figure 1, see section on supplementary data given at the end of this article). Both sites showed vice versa binding to the other corresponding transcription factor. The oligonucleotide containing the sequence of binding site $\mathrm{D}$ showed weak supershifting using antibodies against SP1 (Fig. 3B, Supplementary Figure 1). But there was no supershift if ZBP89 antibodies were applied. Consequently, site D alone does not bind to ZBP89 under these experimental conditions. Site A showed a weak and inconsistent shift band even under constant experimental conditions.

\section{Activation of IRS2 promoter in neuronal cells is repressed} by PI3K

PI3K inhibition using LY294002 in SHSY5Y cells revealed an over twofold activation of the IRS2 promoter luciferase reporter plasmid (Fig. 4A). Therefore, we treated SHSY5Y cells with rising concentrations of LY294002 and performed

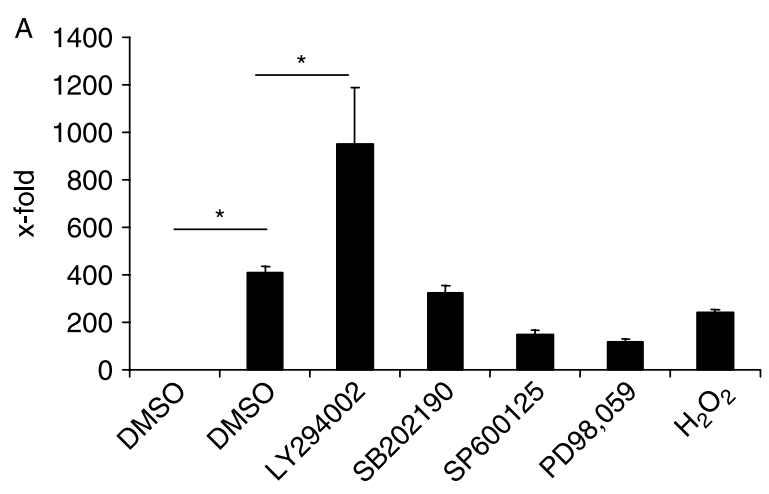

B
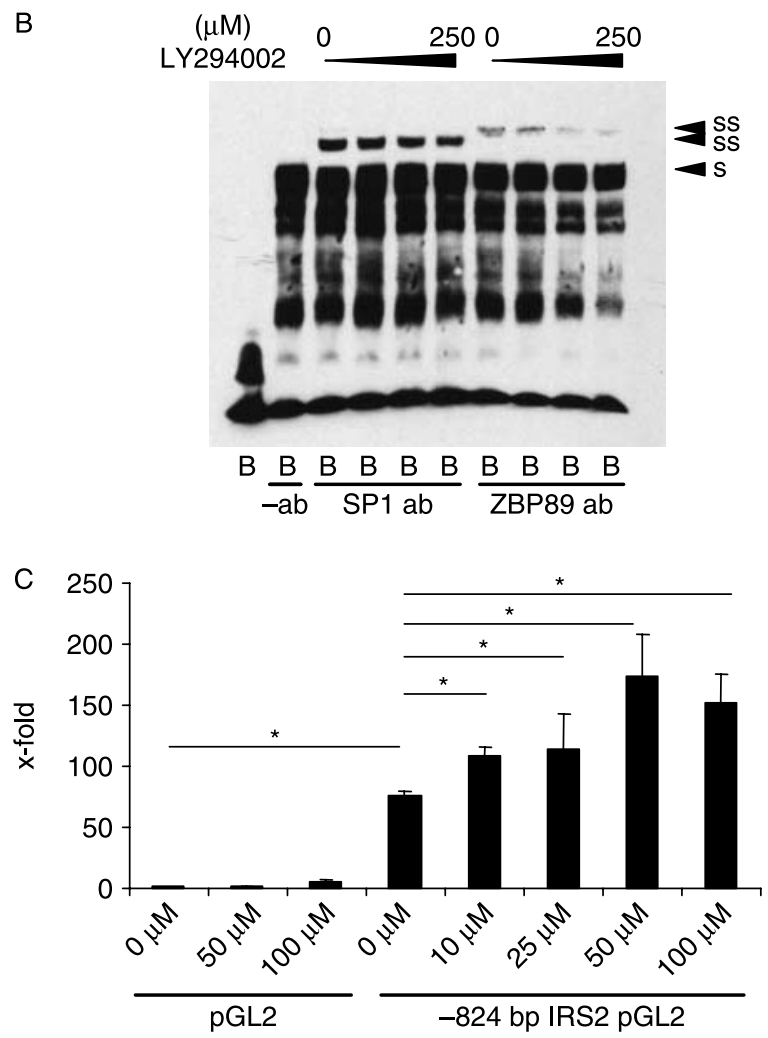

Figure 4 Neuronal IRS2 promoter activity is dependent on PI3K activity. (A) SHSY5Y cells were transfected with the -824 bp-IRS2pGL2 plasmid and dual luciferase assays were performed. Cells were incubated with different inhibitors and substances after transfection as indicated. Quantification represents six independent experiments. Data are means \pm s.D. (B) To reveal the effects of PI3K on ZBP89/SP1 binding to site B, nuclear extracts (NEs) from SHSY5Y cells treated with LY294002 for $30 \mathrm{~h}$ were used in EMSA. Biotinylated double-stranded oligonucleotides representing binding site B were incubated without (lane 1), with nuclear extracts (lane 2), and with nuclear extracts and specific antibodies against SP1 (lanes 3-6) or ZBP89 (lanes 7-10). NEs were taken from cells incubated with rising amounts of LY294002 inhibitor. Shift and supershift bands are indicated by arrows. (C) SHSY5Y cells were transfected with -824 bp-IRS2-pGL2 plasmid as in (A). Cells were incubated with rising concentrations of LY294002. Quantification represents six independent experiments. Data are means \pm s.D. $\left({ }^{*} P<0 \cdot 0005\right.$, unpaired Student's $t$-test). 
EMSA using NEs obtained from these cells. Since binding site $\mathrm{B}$ is of particular relevance for promoter activation in luciferase assays, we used a double-stranded biotinylated oligonucleotide containing the sequence of binding site $\mathrm{B}$ in EMSA experiments. As shown in Fig. 4B, ZBP89 supershift was inhibited by rising LY294002 concentrations in these cells, whereas SP1 binding was unchanged. Thus, PI3K inhibition activates the IRS2 promoter via dissociation of ZBP89 from the activation cassette. We also performed luciferase promoter reporter assays in SHSY5Y cells with rising concentrations of LY294002 (Fig. 4C) revealing a concentration-dependent increase in IRS2 promoter activity after inhibition of PI3K.

Serum deprivation changes ZBP89/SP1 binding within the activation cassette

We starved SHSY5Y cells in serum-free medium from 0 to $48 \mathrm{~h}$ (Fig. 5). Thereafter, NEs were used in EMSA with antibodies against SP1 or ZBP89 for supershift. Transcription activator SP1 showed a stronger supershift band at binding site $\mathrm{B}$ with increasing starvation time (Fig. 5A and C). In the same setting, binding site $\mathrm{C}$ containing oligonucleotides showed decreased SP1 binding (Fig. 5A and C) proving a functional interaction between the different binding sites in the described cassette. However, recruitment of the repressor ZBP89 to the transcriptional complex did not change under conditions of serum deprivation since the supershift band did not change using ZBP89 antibodies and oligonucleotides representing the sequence for binding site $\mathrm{B}$ or $\mathrm{C}$ (Fig. 5B).

\section{Generation of stable ZBP89 knockdown SHSY5Y cells}

Since we found that ZBP89 might be important to build up transcription complexes regulating IRS2 promoter activity in neuronal cells, we aimed to identify the effects of mutations of the ZBP89 binding sites in the promoter. Unfortunately, it is impossible to investigate the function of the ZBP89 binding sites in the IRS2 promoter by mutation of luciferase reporter plasmids without destroying the overlapping SP1 binding sites. Therefore, we decided to use a siRNA-based approach. We cloned two sequences into the pSuppressor Neo vector. One sequence is active in suppressing ZBP89 transcripts (5'-AAGATCGAAGTATGCCTCACCTT- $\left.3^{\prime}\right)$, and for control we used the same sequence mutated in five bps (5'-AAGATCGAACGTGTCCTCACCTT- $\left.3^{\prime}\right)$ which is ineffective in ZBP89 suppression (Bai et al. 2004). The constructs were linearized and transfected into SHSY5Y cells. Stable cell lines were generated as described in the Materials and Methods section.

\section{ZBP89 represses IRS2 promoter activity}

We checked the two cell lines 'SHSY5Y ZBP89 down' and 'SHSY5Y ZBP89 control' for ZBP89 expression. We were able to show downregulation of ZBP89 in the cell line
A

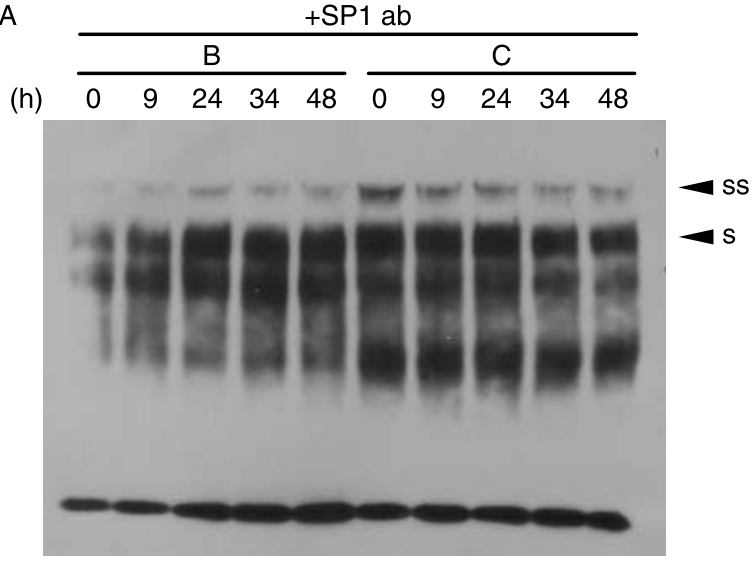

B
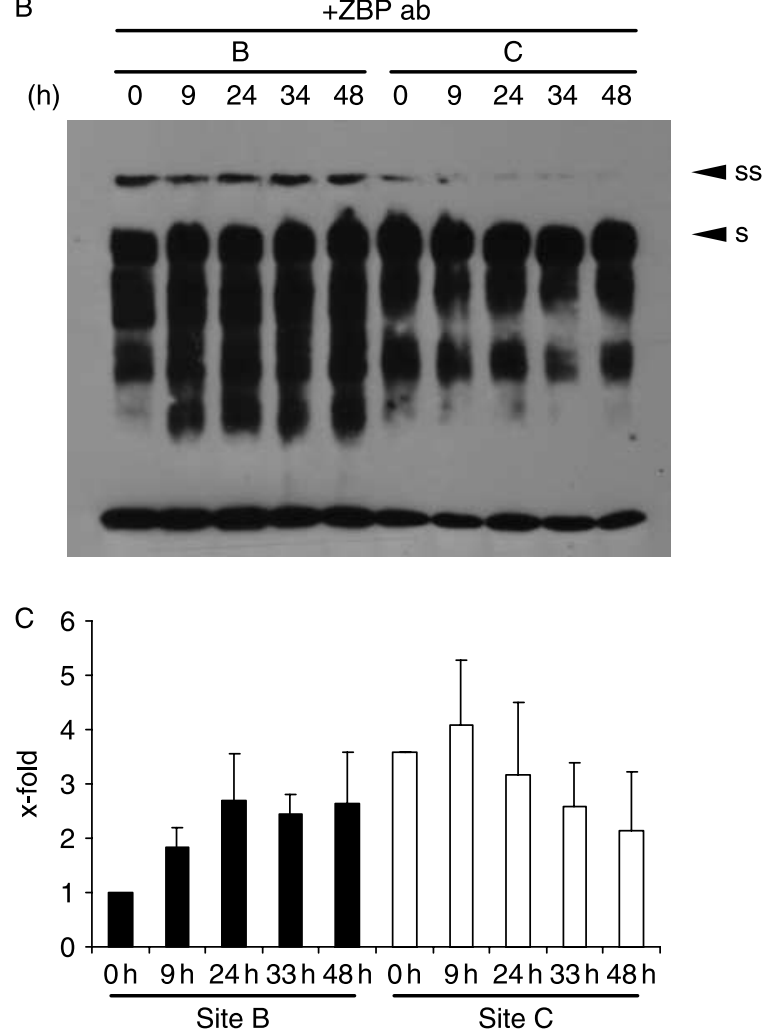

Figure 5 Effect of serum starvation on differential binding of SP1 and ZBP89 to binding sites B and C. SHSY5Y cells were starved for the indicated time points. Afterwards, nuclear extracts were prepared and EMSA was performed. Nuclear extracts were incubated with double-stranded biotinylated oligonucleotides representing binding sites B (lanes 1-5) and C (lanes 6-10). Samples were incubated together with SP1 (A) or ZBP89 (B) antibodies to perform supershift. (C) Quantification represents three independent experiments as shown in (A). Data are means \pm s.D.

expressing the functional siRNA (Fig. 6A). The control cell line showed nearly unaltered ZBP89 expression. IRS2 protein expression was upregulated in SHSY5Y ZBP89 knockdown cells as shown by western blotting (top of Fig. 6A) and 

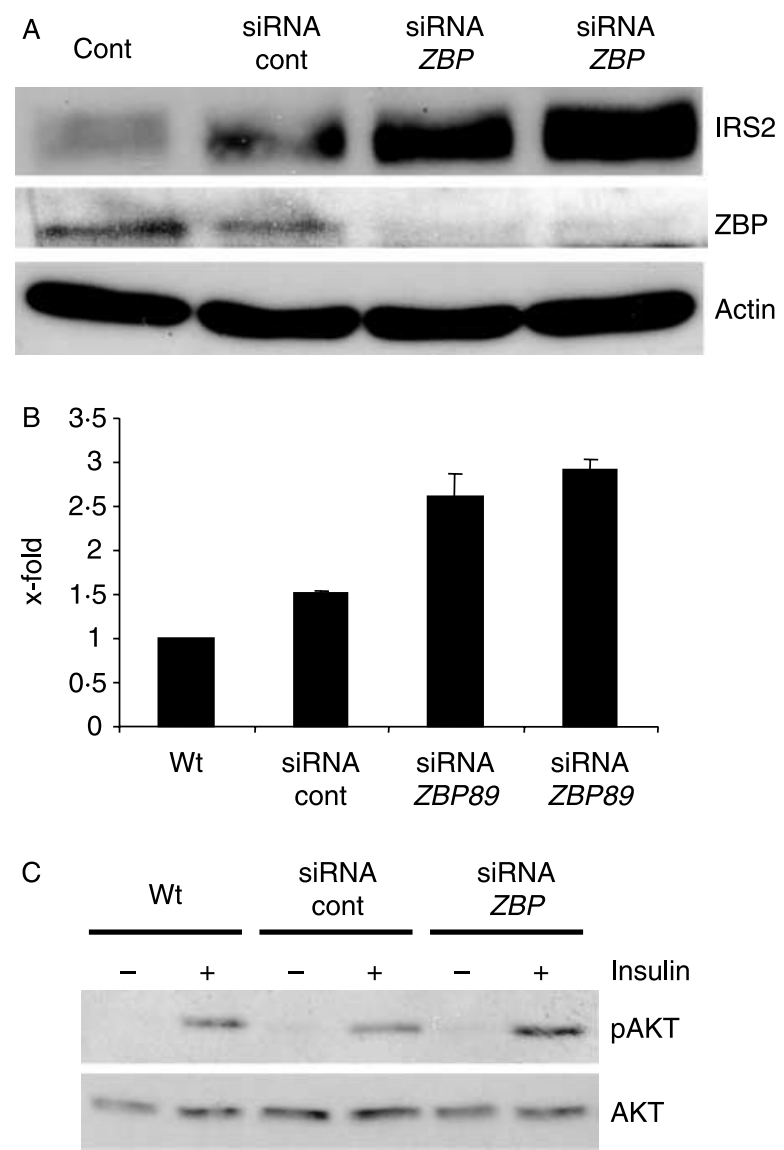

Figure 6 Stable knockdown of ZBP89 in SHSY5Y cells. (A) Cells were generated as described in experimental procedures. For control of ZBP89 expression, total cell lysate was prepared and samples were analyzed using western blot. Wild-type cells, a control cell line stably transfected with a mutant nonfunctional siRNA, and two clones stably transfected with the functional construct are shown. Actin was used for loading control. (B) Same cells were used for Taqman assays. Quantification of four independent experiments is shown. Data are means \pm s.D. (C) Wildtype, control, and ZBP89 downregulated SHSY5Y cells were kept serum free for $30 \mathrm{~h}$. Cells were stimulated with or without $100 \mathrm{nM}$ insulin for $5 \mathrm{~min}$ as indicated. After preparing total cell lysates, western blots were performed using anti-phospho AKT ser ${ }^{473}$ antibodies and anti-AKT antibodies as loading control. Data represent a typical result of four independent experiments.

quantitative real-time PCR (Fig. 6B). In conclusion, ZBP89 works as a repressor in the described regulation cassette of the IRS2 promoter. Loss of the repressor causes activation of IRS2 transcription leading to an about twofold increased IRS2 expression in neuronal cells.

\section{Insulin signaling is increased in ZBP89 knockdown SHSY5Y cells}

We stimulated 'SHSY5Y ZBP89 down' and 'SHSY5Y ZBP89 control' cells with $100 \mathrm{nM}$ insulin after cells were kept serum free for $30 \mathrm{~h}$ (Fig. 6C). After performing
SDS-PAGE using total cell lysates, we were able to detect phosphorylation of AKT at $\mathrm{Ser}^{473}$ in control cells, but AKT phosphorylation was slightly stronger in ZBP89 knockdown cells after insulin stimulation. Consequently, ZBP89 might be able to modulate insulin/IGF1 receptor signaling via the control of IRS2 expression levels in neuronal cells.

\section{Discussion}

Until now, no function for ZBP89 in the regulation of proteins from the insulin/IGF1 receptor signaling cascade has been described. In the recent past, neuronal insulin/IGF1 receptor signaling has been shown to be important not only for the regulation of energy homeostasis but also for aging and longevity (Kenyon et al. 1993, Giannakou \& Partridge 2007, Taguchi et al. 2007, Cohen \& Dillin 2008).

ZBP89 is a transcription factor binding to GC-rich elements in different genes. In 1996, Merchant et al. discovered that EGF-induced activation of the gastrin promoter was repressed by ZBP89. The protein binds to and regulates the vimentin promoter by repressing SP1-mediated activation (Zhang et al. 2003). Furthermore, ZBP89 has been shown to mediate the inhibitory effects of saturated fatty acids on the GH receptor gene (Thimmarayappa et al. 2006). Direct binding of ZBP89 to p53, which causes growth arrest via p53 retention in the nucleus (Bai \& Merchant 2001), has been described as well. Interestingly, ZBP89 induces apoptosis in a JNK-dependent, but p53-independent manner (Bai et al. 2004). In summary, ZBP89 inhibits cell proliferation (Remington et al. 1997), induces apoptosis, and cooperates or competes with SP1 promoter binding sites, for example, in the STAT1 (Bai \& Merchant 2003) and the vimentin promoter (Zhang et al. 2003).

Two types of interactions between SP1 and ZBP89 to regulate promoter activity have been described. In the first case, both proteins compete for binding to an overlapping binding site. In the second case, e.g. in the vimentin promoter, binding sites of both transcription factors are located side by side (Zhang et al. 2003) and interaction of both proteins is achieved by DNA structure or multiprotein complexes. With regard to the IRS2 promoter activation cassette containing four potential ZBP89/SP1 binding sites, both models must be taken into consideration. Interestingly, all constructs containing a mutation annihilating SP1 binding to site $\mathrm{B}$ showed a reduction of luciferase activity. A single mutation on site B alone had a smaller effect on IRS2 promoter activity than combined mutations (including site B) of one, two, or three other sites. We were not able to verify binding of SP1 to all the sites in EMSA, but all the four sites might be essential to fully activate the promoter in neuronal cells under certain conditions. This assumption is supported by the fact that mutating SP1 binding site B in combination with $\mathrm{A}$ or $\mathrm{D}$ has a stronger effect on IRS2 promoter activity than the mutation of site B alone, even though we could only 
detect inconstant binding to site $\mathrm{A}$ and weak binding to site $\mathrm{D}$ under basal conditions. The cassette is arranged in two blocks as shown in Fig. 2B. These blocks are separated by $\sim 20 \mathrm{bp}$ meaning that SP1 (A) and SP1 (B) binding sites are located on the same side of the DNA as ZBP89 (C) and SP1 (D) sites. With regard to the two adjacent binding sites within each block, the ZBP89 and SP1 sites are located directly side by side but do not overlap. Within each binding site, the SP1 and ZBP89 recognition sites overlap, which made it impossible to mutate ZBP89 binding without destroying SP1 binding simultaneously. Therefore, we chose a knockdown approach using siRNA against ZBP89.

In EMSA, we found that there was differential binding of ZBP89 and SP1 to sites B and C. Consequently, we assumed that the regulation of promoter activity might be controlled via competitive binding of the two proteins to each binding site. Furthermore, serum deprivation experiments revealed a possible functional interaction between the different ZBP89/SP1 binding sites as the mechanism of transcriptional regulation of the IRS2 promoter. After inhibition of PI3K, we found a strong activation of the IRS2 promoter in luciferase assays. We speculate that there might be an inhibitory effect of PI3K mediated via ZBP89 on binding site B. In fact, we were able to show that ZBP89 binding to site $\mathrm{B}$ was weakened with increasing PI3K inhibition. In conclusion, PI3K might inhibit the regulation cassette via ZBP89 binding to site B. Furthermore, we found a weak interaction of SP1 with site D and an inconstant binding to site $\mathrm{A}$ as well as an about $50 \%$ decreased promoter activity if site $\mathrm{B}$ is mutated simultaneously, suggesting that binding sites $\mathrm{A}$ and $\mathrm{D}$ are also involved in promoter activation.

However, a repressing effect on SP1 activity by ZBP89 on the bovine adrenodoxin promoter (Cheng et al. 2000) independent of direct ZBP89 binding to the DNA has been described. Concerning the IRS 2 promoter, it might be that the transcriptional complex containing ZBP89 influences SP1 binding or activity of the already bound SP1 at adjacent sites. Recently, a potential mechanism for this has been described (Chupreta et al. 2007). In detail, sumoylation of two motives flanking the DNA-binding domain of ZBP89 inhibited its interaction with SP1. Direct competition for a single individual DNA binding site between ZBP89 and SP1 was not influenced by this modification investigating one single ornithine decarboxylase (ODC) promoter site (Chupreta et al. 2007). But engineered luciferase reporter plasmids bearing multiple units of ODC response elements (ZBP89/SP1, similar to the activation cassette in our work) presented with higher luciferase activities after eliminating ZBP89 sumoylation (Chupreta et al. 2007).

To definitely test the influence of ZBP89 on IRS2 promoter repression, we created ZBP89 knockdown SHSY5Y cells using siRNA against ZBP89. Without changing SP1 concentration in these cells, we achieved a twofold increased IRS2 protein expression just by downregulation of ZBP89. Furthermore, we were able to show twofold higher IRS2 mRNA levels by comparing ZBP89 knockdown to control cells expressing the mutant form of the used hairpin siRNA. To investigate whether downregulation of ZBP89 influences insulin receptor signaling, we performed insulin stimulation experiments using $\mathrm{PKB}$ phosphorylation as readout (Pessin \& Saltiel 2000). PKB phosphorylation was slightly increased after insulin stimulation in ZBP89 knockdown cells than in control cells. Consequently, ZBP89 might be able to change the activity of the insulin/IGF1 receptor signaling cascade via the regulation of IRS2 levels in neuronal cells.

Thus, the present work revealed several new findings: 1) neuronal IRS 2 promoter activity is tightly controlled by an activation cassette composed of four ZBP89/SP1 binding sites, 2) ZBP89 functions as a transcriptional repressor on the IRS2 promoter in neurons, 3) the different ZBP89/SP1 binding sites of the IRS2 promoter show functional interaction, and 4) ZBP89 binding to the IRS2 promoter is regulated by the PI3K pathway.

\section{Supplementary data}

This is linked to the online version of the paper at http://dx.doi.org/10.1677/ JOE-09-0266.

\section{Declaration of interest}

We declare that there is no conflict of interest that could be perceived as prejudicing the impartiality of the research reported.

\section{Funding}

The work was supported by Fritz-Thyssen Foundation (grant number Az. 10.06.2.209 M S) and the Alzheimer Forschungs Initiative e.V. (grant number 08813 M S).

\section{Acknowledgements}

We gratefully thank Moritz M Hettich and Katharina Schilbach for critical reading and discussion of the data.

\section{References}

Bai L \& Merchant JL 2001 ZBP-89 promotes growth arrest through stabilization of p53. Molecular and Cellular Biology 21 4670-4683.

Bai L \& Merchant JL 2003 Transcription factor ZBP-89 is required for STAT1 constitutive expression. Nucleic Acids Research 31 7264-7270.

Bai L, Yoon SO, King PD \& Merchant JL 2004 ZBP-89-induced apoptosis is p53-independent and requires JNK. Cell Death and Differentiation 11 $663-673$.

Burks DJ, Font de Mora J, Schubert M, Withers DJ, Myers MG, Towery HH, Altamuro SL, Flint CL \& White MF 2000 IRS-2 pathways integrate female reproduction and energy homeostasis. Nature 407 377-382.

Cheng PY, Kagawa N, Takahashi Y \& Waterman MR 2000 Three zinc finger nuclear proteins, Sp1, Sp3, and a ZBP-89 homologue, bind to the cyclic adenosine monophosphate-responsive sequence of the bovine adrenodoxin gene and regulate transcription. Biochemistry 39 4347-4357.

Journal of Endocrinology (2010) 204, 199-208 
Chupreta S, Brevig H, Bai L, Merchant JL \& Iniguez-Lluhi JA 2007 Sumoylation-dependent control of homotypic and heterotypic synergy by the Kruppel-type zinc finger protein ZBP-89. Journal of Biological Chemistry 282 36155-36166.

Cohen E \& Dillin A 2008 The insulin paradox: aging, proteotoxicity and neurodegeneration. Nature Reviews. Neuroscience 9 759-767.

Dignam JD, Lebovitz RM \& Roeder RG 1983 Accurate transcription initiation by RNA polymerase-II in a soluble extract from isolated mammalian nuclei. Nucleic Acids Research 11 1475-1489.

Dong XC, Copps KD, Guo S, Li Y, Kollipara R, DePinho RA \& White MF 2008 Inactivation of hepatic Foxo1 by insulin signaling is required for adaptive nutrient homeostasis and endocrine growth regulation. Cell Metabolism 8 65-76.

Giannakou ME \& Partridge L 2007 Role of insulin-like signalling in Drosophila lifespan. Trends in Biochemical Sciences 32 180-188.

Haeusler RA \& Accili D 2008 The double life of Irs. Cell Metabolism 8 7-9.

Ide T, Shimano H, Yahagi N, Matsuzaka T, Nakakuki M, Yamamoto T, Nakagawa Y, Takahashi A, Suzuki H, Sone H et al. 2004 SREBPs suppress IRS-2-mediated insulin signalling in the liver. Nature Cell Biology 6 351-357.

Kenyon C, Chang J, Gensch E, Rudner A \& Tabtiang R 1993 A C. elegans mutant that lives twice as long as wild type. Nature 366 461-464.

Kubota N, Kubota T, Itoh S, Kumagai H, Kozono H, Takamoto I, Mineyama T, Ogata H, Tokuyama K, Ohsugi M et al. 2008 Dynamic functional relay between insulin receptor substrate 1 and 2 in hepatic insulin signaling during fasting and feeding. Cell Metabolism 8 49-64.

Lavan BE, Fantin VR, Chang ET, Lane WS, Keller SR \& Lienhard GE 1997 a A novel $160-\mathrm{kD}$ a phosphotyrosine protein in insulin-treated embryonic kidney cells is a new member of the insulin receptor substrate family. Journal of Biological Chemistry 272 21403-21407.

Lavan BE, Lane WS \& Lienhard GE $1997 b$ The 60-kDa phosphotyrosine protein in insulin-treated adipocytes is a new member of the insulin receptor substrate family. Journal of Biological Chemistry 272 11439-11443.

Law GL, Itoh H, Law DJ, Mize GJ, Merchant JL \& Morris DR 1998 Transcription factor ZBP-89 regulates the activity of the ornithine decarboxylase promoter. Journal of Biological Chemistry 273 19955-19964.

Lin X, Taguchi A, Park S, Kushner JA, Li F, Li Y \& White MF 2004 Dysregulation of insulin receptor substrate 2 in beta cells and brain causes obesity and diabetes. Journal of Clinical Investigation 114 908-916.

Merchant JL, Du M \& Todisco A 1999 SP1 phosphorylation by ERK2 stimulates DNA binding. Biochemical and Biophysical Research Communications 254 454-461.

Nakagawa Y, Shimano H, Yoshikawa T, Ide T, Tamura M, Furusawa M, Yamamoto T, Inoue N, Matsuzaka T, Takahashi A et al. 2006 TFE3 transcriptionally activates hepatic IRS-2, participates in insulin signaling and ameliorates diabetes. Nature Medicine 12 107-113.

Pessin JE \& Saltiel AR 2000 Signaling pathways in insulin action: molecular targets of insulin resistance. Journal of Clinical Investigation 106 165-169.

Pfaffl MW 2001 A new mathematical model for relative quantification in real-time RT-PCR. Nucleic Acids Research 29 e45.

Remington MC, Tarle SA, Simon B \& Merchant JL 1997 ZBP-89, a Kruppel-type zinc finger protein, inhibits cell proliferation. Biochemical and Biophysical Research Communications 237 230-234.
Rivera EJ, Goldin A, Fulmer N, Tavares R, Wands JR \& de la Monte SM 2005 Insulin and insulin-like growth factor expression and function deteriorate with progression of Alzheimer's disease: link to brain reductions in acetylcholine. Journal of Alzheimer's Disease 8 247-268.

Schubert M, Brazil DP, Burks DJ, Kushner JA, Ye J, Flint CL, Farhang-Fallah J, Dikkes P, Warot XM, Rio C et al. 2003 Insulin receptor substrate-2 deficiency impairs brain growth and promotes tau phosphorylation. Journal of Neuroscience 23 7084-7092.

Shimano H 2007 SREBP-1c and TFE3, energy transcription factors that regulate hepatic insulin signaling. Journal of Molecular Medicine 85 437-444.

Steen E, Terry BM, Rivera EJ, Cannon JL, Neely TR, Tavares R, Xu XJ, Wands JR \& de la Monte SM 2005 Impaired insulin and insulin-like growth factor expression and signaling mechanisms in Alzheimer's disease - is this type 3 diabetes? Journal of Alzheimer's Disease 7 63-80.

Sun XJ, Rothenberg P, Kahn CR, Backer JM, Araki E, Wilden PA, Cahill DA, Goldstein BJ \& White MF 1991 Structure of the insulin receptor substrate IRS-1 defines a unique signal transduction protein. Nature 352 73-77.

Sun XJ, Wang LM, Zhang Y, Yenush L, Myers MG Jr, Glasheen E, Lane WS, Pierce JH \& White MF 1995 Role of IRS-2 in insulin and cytokine signalling. Nature 377 173-177.

Taguchi A, Wartschow LM \& White MF 2007 Brain IRS2 signaling coordinates life span and nutrient homeostasis. Science 317 369-372.

Thimmarayappa J, Sun J, Schultz LE, Dejkhamron P, Lu C, Giallongo A, Merchant JL \& Menon RK 2006 Inhibition of growth hormone receptor gene expression by saturated fatty acids: role of Kruppel-like zinc finger factor, ZBP-89. Molecular Endocrinology 20 2747-2760.

Thirone AC, Huang C \& Klip A 2006 Tissue-specific roles of IRS proteins in insulin signaling and glucose transport. Trends in Endocrinology and Metabolism 17 72-78.

Wang Y, Kobori JA \& Hood L 1993 The ht beta gene encodes a novel CACCC box-binding protein that regulates T-cell receptor gene expression. Molecular and Cellular Biology 13 5691-5701.

White MF 2003 Insulin signaling in health and disease. Science 302 1710-1711.

Wieczorek E, Lin Z, Perkins EB, Law DJ, Merchant JL \& Zehner ZE 2000 The zinc finger repressor, ZBP-89, binds to the silencer element of the human vimentin gene and complexes with the transcriptional activator, Sp1. Journal of Biological Chemistry 275 12879-12888.

Withers DJ, Burks DJ, Towery HH, Altamuro S, Flint C \& White MF 1999 Irs-2 coordinates Igf-1 receptor-mediated beta-cell development and peripheral insulin signalling. Nature Genetics 23 32-40.

Zhang J, Ou J, Bashmakov Y, Horton JD, Brown MS \& Goldstein JL 2001 Insulin inhibits transcription of IRS-2 gene in rat liver through an insulin response element (IRE) that resembles IREs of other insulin-repressed genes. PNAS 98 3756-3761.

Zhang X, Diab IH \& Zehner ZE 2003 ZBP-89 represses vimentin gene transcription by interacting with the transcriptional activator, Sp1. Nucleic Acids Research 31 2900-2914.

Received in final form 19 October 2009

Accepted 29 October 2009

Made available online as an Accepted Preprint 29 October 2009 